\title{
High Serum Levels of iNOS and MIP-I $\alpha$ are Associated with Post-Stroke Depression
}

\author{
Xiuli Wang ${ }^{1,2, *}$ \\ Cui Fang ${ }^{1,2, *}$ \\ Xiaoxi Liu ${ }^{1, *}$ \\ Wei Wei ${ }^{3}$ \\ Mengfan Zhang ${ }^{2,4}$ \\ Shuang Chen ${ }^{2,4}$ \\ Fuping $\mathrm{Shi}^{1}$
}

'Department of Neurology, Affiliated Hospital of Hebei University, Baoding, People's Republic of China; ${ }^{2}$ School of Clinical Medicine, Hebei University, Baoding, People's Republic of China; ${ }^{3}$ Department of Radiology, Hebei Province Veterans Hospital, Baoding, People's Republic of China; ${ }^{4}$ Department of Internal Medicine, Affiliated Hospital of Hebei University, Baoding, People's

Republic of China

*These authors contributed equally to this work
Correspondence: Fuping Shi Department of Neurology, Affiliated Hospital of Hebei University, No. 212 East Yuhua Road, Baoding, Hebei, 071000,

People's Republic of China

Tel +86 I32 732I 0990

Email sfp-1000@163.com
Purpose: Post-stroke depression (PSD) is one of the emotional disorders after the onset of stroke. Many studies have indicated that inflammatory processes can promote the occurrence and development of PSD. The purpose of our study was to explore the potential relationship between PSD and two inflammatory biomarkers: inducible nitric oxide synthase (iNOS) and macrophage inflammatory protein $1 \alpha$ (MIP-1 $\alpha)$.

Methods: In total, 80 patients diagnosed with depression after the first-ever acute ischemic stroke were enrolled in PSD group consecutively. During the same period, 40 non-depressed patients following the first-ever acute ischemic stroke and 40 healthy control subjects were recruited as non-PSD group and normal group, respectively. All participants have performed serum iNOS and MIP-1 $\alpha$ level examination with enzyme-linked immunosorbent assay (ELISA). The severity of depressive symptoms was evaluated by the 24-item Hamilton Depression Scale (HAMD-24).

Results: Serum iNOS and MIP-1 $\alpha$ levels were significantly higher in PSD group than those in non-PSD and normal groups $(P<0.001)$. Serum iNOS and MIP-1 $\alpha$ levels of PSD patients with varying degrees of depression were significantly different, serum iNOS and MIP- $1 \alpha$ levels became higher as the depressive symptoms became more severe $(P<0.05)$. There was a positive correlation between the elevated levels of iNOS, MIP-1 $\alpha$ and HAMD scores $(r=$ $0.262,0.209, P<0.05)$. Logistic regression analysis showed that both serum iNOS and MIP$1 \alpha$ levels were independently associated with PSD (OR $=2.790,95 \%$ CI: $0.712-10.933, P<$ 0.05 and $\mathrm{OR}=1.922,95 \% \mathrm{CI}: 0.648-9.815, P<0.05$, respectively) after adjustment for possible relevant confounders.

Conclusion: High serum levels of iNOS and MIP-1 $\alpha$ were found to be associated with the development of PSD and closely related to its severity.

Keywords: post-stroke depression, inducible nitric oxide synthase, macrophage inflammatory protein $1 \alpha$, HAMD scores

\section{Introduction}

Stroke has become the third disabling and the second mortal disease around the world. ${ }^{1}$ In recent years, although great progress has been made in the prevention and treatment of stroke, it remains an urgent problem to be solved due to its high recurrence rate and serious complications. ${ }^{2,3}$ Stroke survivors tend to be left with different degrees of physical and psychiatric disabilities. ${ }^{4}$ In recent years, psychic syndrome after stroke has drawn increasing attention, such as post-stroke depression (PSD), more than one-third of stroke survivors experience varying degrees of depressive symptoms at any time during follow-up. ${ }^{5}$ The main clinical symptoms of PSD include somnipathy, depression, anxiety and even suicide. ${ }^{6}$ The etiology and 
pathogenesis of PSD are multifactorial and have not been fully understood. ${ }^{7}$ Therefore, it is of great significance to reveal more detailed pathophysiological mechanisms and find more convincing biological markers about PSD.

Previous studies have found that the L-arginine-NOcyclic guanosine monophosphate (cGMP) signaling pathway participates in the pathogenesis of mood disorders including depression. ${ }^{8}$ As a signal molecule, nitric oxide (NO) can be synthesized by three isoforms of nitric oxide synthases (NOSs) and can regulate behavioral, cognitive and emotional processes under normal conditions. ${ }^{9}$ Of the three isoforms of NOSs, inducible nitric oxide synthase (iNOS) is mainly involved in the production of NO under inflammatory conditions and particularly relevant to neuropsychiatric disorders. ${ }^{9}$ Some studies have suggested that iNOS can induce the occurrence and development of depression through neuroinflammatory signaling pathways. ${ }^{10}$ In addition, some investigations have confirmed that the expression of iNOS was increased in depressed patients. ${ }^{11,12}$ Moreover, a study suggested that injecting iNOS inhibitor aminoguanidine into one side of the hippocampus significantly inhibited depression-like behaviors induced by chronic unpredictable mild stress (CUMS). ${ }^{13}$ Another study found that after antidepressant treatment in a vivo model of depression, the production of NO was decreased by inhibiting the expression of iNOS. ${ }^{14}$ The possible mechanism is that inhibiting iNOS signaling pathway may alleviate depressive symptoms by exerting anti-inflammatory and neuroprotective effects. ${ }^{15}$

Macrophage inflammatory protein $1 \alpha$ (MIP-1 $\alpha$ ) belongs to a member of the $\mathrm{CC}$ subfamily of chemokines and its release is considered a crucial process in the recruitment of inflammatory cells during initiation and subsequent maintenance of inflammatory responses. ${ }^{16}$ It has been suggested that MIP-1 $\alpha$ may be involved in the development of depressive symptoms through the recruitment of inflammatory cells. ${ }^{17}$ Besides, iNOS can regulate the production of MIP$1 \alpha$. After inducing the production of NO in the wild-type mice, the levels of MIP-1 $\alpha$ was increased, but for the iNOS gene knockout mice, the release of MIP-1 $\alpha$ was not affected. ${ }^{18}$ Moreover, the expression of MIP-1 $\alpha$ was significantly inhibited by NOS inhibitor. ${ }^{19}$ Based on the above evidence, iNOS and MIP-1 $\alpha$ may jointly lead to the occurrence of depressive symptoms. Nevertheless, the specific mechanism of the influence of iNOS and MIP-1 $\alpha$ on PSD is still unclear.

Therefore, the main aim of our study was to explore whether the elevated levels of iNOS and MIP-1 $\alpha$ can promote the development of PSD in Chinese subjects so as to provide a specific basis for the future research of PSD.

\section{Methods}

\section{Study Subjects}

From October 2017 to January 2019, 80 patients diagnosed with depression following the first-ever acute ischemic stroke in the basal ganglia were enrolled in this study. During the same period, 40 non-depressed patients after the first-ever acute ischemic stroke in the basal ganglia and 40 healthy control subjects were screened consecutively. This study was performed in the Affiliated Hospital of Hebei University. Inclusion criteria: (1) age 36-70 years; (2) evidence of cerebrovascular disease was present before or at the time of the first onset of depressive symptoms and patients were admitted to hospital within 3 days; (3) acute ischemic stroke was diagnosed with a cranial computed tomography (CT) or magnetic resonance imaging (MRI) examination on admission; (4) patients had clear consciousness, cooperative physical examination, no mental retardation and aphasia. Exclusion criteria: (1) significant neurological illnesses other than stroke; (2) a previous history of psychiatric disorders or psychiatric treatment; (3) severe infection or organ damage such as heart, liver or kidney failure; (4) unable to cooperate with related examination due to various reasons. This is an observational clinical study approved by the Ethics Committee of the Affiliated Hospital of Hebei University (approved number: 201722). Our study was performed in accordance with the ethical guidelines of the 1975 Declaration of Helsinki. All subjects provided written informed consent.

\section{Baseline Information Collection}

Demographic characteristics (sex, age, smoking and drinking status, hypertension, type II diabetes, arrhythmia, cerebral arteriosclerosis and lipid metabolism disorder) of all participants were recorded. The neurological function of all patients with acute ischemic stroke was assessed by the National Institutes of Health Stroke Scale (NIHSS) on admission. $^{20}$

\section{Assessment of Depression}

The 24-item Hamilton Depression Scale (HAMD-24) was used to evaluate depression of all subjects. Patients with a HAMD score $>7$ were diagnosed with PSD according to the Diagnostic and Statistical Manual of Mental Disorders 
(5th edition) (DSM-V) ${ }^{21}$ The 80 patients diagnosed with depression after the first-ever acute ischemic stroke were enrolled in PSD group. The PSD group was divided into mild PSD group (8-16), moderate PSD group (17-23) and severe PSD group $(\geq 24)$ according to HAMD scores. ${ }^{22}$ During the same period, 40 non-depressed patients after the first-ever acute ischemic stroke and 40 healthy control subjects were recruited as non-PSD group and normal group, respectively.

\section{Serum Sample Collection, Storage, and Measurements}

For all participants, $4 \mathrm{~mL}$ fasting blood samples were drawn, let them stand for 30 minutes at room temperature and then centrifuged (3000 rpm, $10 \mathrm{~min})$. The serum was separated and stored at $-80^{\circ} \mathrm{C}$ immediately before the assays, avoid repeated freezing and thawing. The serum iNOS, MIP-1 $\alpha$ concentrations were measured using a commercially available enzyme-linked immunosorbent assay (ELISA) (Bio-Techne China Co., Ltd. and Abcam, Trading Co., Ltd, Shanghai, China, respectively). The steps were carried out strictly in accordance with instructions.

\section{Statistical Methods}

Statistical analysis was performed with SPSS version 19.0 software (SPSS Inc., Chicago, IL, USA). Continuous normal distribution measurement data were expressed as mean \pm standard deviation (SD), enumeration data were expressed as cases (n) or percentage (\%). The Chi-square $\left(x^{2}\right)$ test was used for the comparison of enumeration data, $t$-test or analysis of variance was used for the comparison of measurement data. The correlation between HAMD scores and the levels of serum iNOS and MIP-1 $\alpha$ was analyzed by the Pearson correlation coefficient analysis. After adjustment for potential confounders, stepwise binary logistic regression was used to identify predictors of occurrence of PSD, potential confounding factors were selected based on previously identified risk factors. $P<$ 0.05 was regarded as statistically significant in two-tailed tests.

\section{Results Baseline Characteristics}

In this study, 80 PSD patients with an average age of 54.7 \pm 11.36 years and 40 non-depressed patients after stroke with an average age of $53.8 \pm 10.93$ years constituted the PSD group and non-PSD group, respectively. The general clinical situations of all patients with acute ischemic stroke are shown in Table 1. No significant differences were found between depressed patients and non-depressed patients after acute ischemic stroke with regard to sex, age, NIHSS scores, smoking and drinking status, hypertension, type II diabetes, arrhythmia, cerebral arteriosclerosis and lipid metabolism disorder (all $P>0.05$ ).

\section{Relationship Between Serum iNOS and MIP-I $\alpha$ Levels and PSD}

Our study investigated the difference in serum iNOS and MIP- $1 \alpha$ levels among PSD group, non-PSD group and normal group. Serum iNOS and MIP- $1 \alpha$ levels were significantly higher in PSD group than those in non-PSD group $(F=289.41,62.49, P<0.001)$. Compared with the normal group, serum iNOS and MIP-1 $\alpha$ levels of the

Table I Clinical Baseline Characteristics of PSD and Non-PSD Patients

\begin{tabular}{|c|c|c|c|c|}
\hline Variables & $\begin{array}{c}\text { Non-PSD Group } \\
(\mathbf{N}=\mathbf{4 0})\end{array}$ & $\begin{array}{l}\text { PSD Group } \\
(\mathbf{N}=\mathbf{8 0})\end{array}$ & $x^{2} / t$ & $P$-value \\
\hline Sex (male/female) & $23 / 17$ & $46 / 44$ & 0.454 & 0.501 \\
\hline Age (years, mean $\pm S D$ ) & $54.7 \pm 11.36$ & $53.8 \pm 10.93$ & 1.061 & 0.125 \\
\hline Smokers (yes/no) & $17 / 23$ & $31 / 49$ & 0.156 & 0.963 \\
\hline Alcohol consumers (yes/no) & $12 / 28$ & $28 / 52$ & 0.300 & 0.584 \\
\hline NIHSS score $(>5 / \leq 5)$ & $16 / 24$ & $30 / 50$ & 0.079 & 0.791 \\
\hline Hypertension (yes/no) & $29 / 11$ & $53 / 27$ & 0.481 & 0.488 \\
\hline Type II diabetes (yes/no) & $16 / 24$ & $19 / 61$ & 3.408 & 0.065 \\
\hline Arrhythmia (yes/no) & $8 / 32$ & $10 / 70$ & 1.176 & 0.278 \\
\hline Cerebral arteriosclerosis (yes/no) & $14 / 26$ & $27 / 53$ & 0.019 & 0.892 \\
\hline Lipid metabolism disorder (yes/no) & $12 / 28$ & $29 / 51$ & 0.463 & 0.496 \\
\hline
\end{tabular}

Notes: Measurement data were expressed as mean \pm SD, enumeration data were expressed as cases $(n)$. $P<0.05$ indicated statistical significance.

Abbreviations: NIHSS, National Institutes of Health Stroke Scale; PSD, post-stroke depression. 
non-PSD group were higher, but there was no statistical significance $(P>0.05)$. As shown in Table 2.

\section{Relationship Between Serum iNOS and MIP-I $\alpha$ Levels and HAMD Scores}

The 80 patients in PSD group comprised 34 with symptoms of mild depression (42.5\%), 27 with moderate depression (33.75\%), and 19 with severe depression (23.75\%). The HAMD scores of PSD patients were ranged from 8 to 35 and the average score was $21.05 \pm 2.59$. Serum iNOS and MIP- $1 \alpha$ concentrations of PSD patients with varying degrees of depression were significantly different $(F=43.27,4.91$, $P<0.05)$. The severe PSD group had significantly higher levels of iNOS and MIP- $1 \alpha$ than the moderate and mild PSD groups $(P<0.05)$. Compared with the mild PSD group, serum iNOS and MIP-1 $\alpha$ levels of the moderate PSD group were higher $(P<0.05)$. As shown in Table 3 .

We further investigated whether there was a correlation between serum iNOS and MIP-1 $\alpha$ levels and the severity of depression. Pearson correlation coefficient analysis

Table 2 Comparison of the Serum iNOS and MIP-I $\alpha$ Levels of PSD Group, Non-PSD Group and Normal Group

\begin{tabular}{|l|c|c|}
\hline Groups & iNOS (U/mL) & MIP-I $\alpha(\mathbf{n g} / \mathbf{L})$ \\
\hline Normal group $(\mathrm{N}=40)$ & $5.54 \pm 0.86$ & $10.02 \pm 1.55$ \\
Non-PSD group $(\mathrm{N}=40)$ & $16.79 \pm 2.70^{\mathrm{a}}$ & $24.75 \pm 7.72^{\mathrm{a}}$ \\
PSD group $(\mathrm{N}=80)$ & $21.65 \pm 2.93^{\mathrm{ab}}$ & $29.44 \pm 9.70^{\mathrm{ab}}$ \\
\hline F value & 289.41 & 62.49 \\
$P$ value & $<0.001$ & $<0.001$ \\
\hline
\end{tabular}

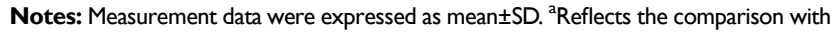
normal group, $P<0.05$ and ${ }^{b}$ reflects the comparison with non-PSD group, $P<0.05$. Abbreviations: PSD, post-stroke depression; iNOS, inducible nitric oxide synthase; MIP-I $\alpha$, macrophage inflammatory protein $\mid \alpha$.

Table 3 Comparison of the Serum iNOS and MIP-I $\alpha$ Levels of PSD Patients with Different Severity of Depression

\begin{tabular}{|l|c|c|}
\hline Groups & iNOS $(\mathbf{U} / \mathbf{m L})$ & MIP- $\mid \boldsymbol{\alpha}(\mathbf{n g} / \mathbf{L})$ \\
\hline Mild PSD group $(\mathrm{N}=34)$ & $18.31 \pm 2.17$ & $26.14 \pm 10.38$ \\
Moderate PSD group $(\mathrm{N}=27)$ & $22.98 \pm 3.24^{\mathrm{a}}$ & $29.81 \pm 9.58^{\mathrm{a}}$ \\
Severe PSD group $(\mathrm{N}=19)$ & $25.73 \pm 3.60^{\mathrm{ab}}$ & $34.82 \pm\left. 8.5\right|^{\mathrm{ab}}$ \\
\hline F value & 43.27 & $4.9 \mid$ \\
$P$ value & $<0.05$ & $<0.05$ \\
\hline
\end{tabular}

Notes: Measurement data were expressed as mean \pm SD. ${ }^{a}$ Reflects the comparison with mild PSD group, $P<0.05$ and ${ }^{\mathrm{b}}$ Reflects the comparison with moderate PSD group, $P<0.05$.

Abbreviations: iNOS, inducible nitric oxide synthase; MIP-I $\alpha$, macrophage inflammatory protein I $\alpha$; PSD, post-stroke depression. confirmed that HAMD scores were positively related to serum iNOS and MIP-1 $\alpha$ levels $(r=0.262,0.209, P<0.05)$.

\section{A Logistic Regression Analysis for Depression in Stroke Patients}

After adjustment for the sex, age, current smoking and drinking status, NHISS scores, hypertension, type II diabetes, arrhythmia, cerebral arteriosclerosis and lipid metabolism disorder, logistic regression analysis showed that iNOS was an independent predictor of PSD with an adjusted OR of $2.790 \quad(95 \%$ CI: $0.712-10.933$, $P<0.05)$. In addition, MIP- $1 \alpha$, was also taken as a continuous variable, can independently predict the development of depression after acute ischemic stroke with an adjusted OR of 1.922 (95\% CI: $0.648-9.815$, $P<0.05)$. As shown in Table 4.

\section{Discussion}

There were some important findings of our study. First, compared with subjects without depression, serum iNOS and MIP- $1 \alpha$ levels were increased significantly in the PSD patients. Second, serum iNOS and MIP-1 $\alpha$ levels differed significantly between PSD patients with different degrees of depression, the levels of iNOS and MIP- $1 \alpha$ became higher as the depressive symptoms became more severe. Third, a positive association was found between serum iNOS, MIP-1 $\alpha$ levels and HAMD scores. Finally, the levels of iNOS and MIP-1 $\alpha$ were independently associated with PSD. Overall, this study indicated that elevated iNOS and MIP-1 $\alpha$ levels were associated with the development of PSD, measuring serum iNOS and MIP$1 \alpha$ levels maybe have a promising evaluation value for PSD.

PSD is one of the emotional disorders after the onset of stroke and predicts poor outcomes of stroke. ${ }^{23}$ In recent years, many studies have shown that inflammation responses play a crucial role in the pathophysiology of PSD, the expression of inflammatory markers was increased in the depressive disorders after stroke. ${ }^{24}$ It has been found that both iNOS and MIP-1 $\alpha$ play a vital role in the occurrence of depressive disorders and the levels of them were increased. ${ }^{25,26}$ However, there is no direct evidence to confirm the association between iNOS and MIP-1 $\alpha$ levels and depression after acute stroke. Our study filled this gap and found PSD patients had higher iNOS and MIP-1 $\alpha$ levels than non-PSD patients as well as normal controls. 
Table 4 Stepwise Logistic Regression to Find Predictors of PSD

\begin{tabular}{|l|c|c|c|c|c|c|c|}
\hline & $\beta$ & S.E. & Wald & P-value & OR & \multicolumn{2}{|c|}{$95 \%$ CI } \\
\cline { 4 - 8 } & & & & & & Lower & Upper \\
\hline Constant & -6.816 & 1.336 & 2.684 & 0.012 & 0.053 & - & 10.933 \\
iNOS $(\mathrm{U} / \mathrm{mL})$ & 1.026 & 0.697 & 6.168 & $0.015^{*}$ & 2.790 & 0.712 & 9.815 \\
MIP-I $\alpha(\mathrm{ng} / \mathrm{L})$ & 0.925 & 0.693 & 4.781 & $0.042^{*}$ & 1.922 & 0.648 & \\
\hline
\end{tabular}

Notes: $* P<0.05$ is considered significant.

Abbreviations: NIHSS, National Institutes of Health Stroke Scale; iNOS, inducible nitric oxide synthase; MIP-I $\alpha$, macrophage inflammatory protein I $\alpha$; OR, odds ratio.

The primary mechanism about increased iNOS levels affecting the occurrence and development of PSD is that iNOS can induce excess NO production which can produce neurotoxicity under the action of reactive oxygen species (ROS) and reactive nitrogen species (RNS). ${ }^{27,28}$ Excess NO can further damage brain tissue and inactivate some enzymes in the brain, causing damage to DNA and mitochondria in cells, thereby blocking DNA replication and energy synthesis in cells. Besides, it can promote the inflammatory responses associated with depression and up-regulate the expression of inflammatory cytokines. ${ }^{29,30}$ Moreover, inflammatory cytokines can induce iNOS gene expression and the production of NO. ${ }^{31-33}$ On the other hand, under the condition of the low production of biogenic amines (such as serotonin and dopamine, both of them are associated with emotion regulation), iNOS can produce more ROS and RNS which can further aggravate the depressive symptoms. ${ }^{34}$

As a hippocampal neuromodulator, MIP-1 $\alpha$ can regulate synaptic plasticity mechanisms involved in learning and memory functions. The concentration of MIP- $1 \alpha$ in normal brain tissue is very low. Under the condition of ischemia and hypoxia, MIP- $1 \alpha$ is increased and mainly comes from microglia cells which are the most significant number of white blood cells in brain tissue during ischemic brain injury. ${ }^{35,36}$ Stroke is a kind of ischemia and hypoxia disease, our study found that the MIP- $1 \alpha$ levels of stroke patients without depression were also elevated compared with healthy subjects. Based on this result, we analyzed the association between MIP- $1 \alpha$ and PSD. In addition to inducing cell migration, MIP- $1 \alpha$ can induce the secretion of TNF-a, IL-1 and IL- $6 .{ }^{17}$ Just like iNOS, MIP-1 $\alpha$ may also interact with these inflammatory cytokines and influence depressive mood in stroke patients. With regard to clinical practice, several studies found that MIP-1 $\alpha$ levels became higher as the depressive symptoms became more severe. ${ }^{37,38}$ Another research suggested that the levels of MIP-1 $\alpha$ were increased in depressed patients with cognitive disorders. ${ }^{26}$ Our results were compatible with those of the above report.

Logistic regression analysis showed that both serum iNOS and MIP-1 $\alpha$ levels were a significant independent predictor of PSD after adjustment for underlying confounding factors, this result further confirmed our speculation. So we can know that iNOS and MIP-1 $\alpha$ may be another two potential biological markers except for BDNF, 5-HT, IL, TNF involved in the pathogenesis of PSD. ${ }^{39-42}$

This study had a few limitations. Firstly, the patients with disturbance of consciousness, mental retardation or other severe situations were not included after stroke, this might lead to some errors in our study results. Secondly, depression was evaluated only one method, so the assessment of depressive symptoms may be partially limited. Finally, serum iNOS and MIP-1 $\alpha$ were evaluated only once, so detecting the serum iNOS and MIP-1 $\alpha$ levels by stages during the follow-up period, we could better observe the changes of inflammatory markers in PSD patients.

\section{Conclusion}

Overall, our study indicated that high serum levels of iNOS and MIP-1 $\alpha$ were associated with PSD and closely related to the severity of depression after acute stroke, so stroke patients should be monitored for high iNOS and MIP-1 $\alpha$ levels and followed up for appropriate interventions. However, whether iNOS and MIP-1 $\alpha$ can influence the occurrence and development of depression after stroke through their interaction with each other still needs further basic research and multicenter controlled study with expanded clinical samples, which may provide new ideas for the further study of PSD.

\section{Acknowledgments}

The authors would like to acknowledge the patients and their families for participating in this study. The authors are grateful to the Affiliated Hospital of Hebei University for helpful comments and feedback throughout this study. 


\section{Funding}

This study was supported by the S\&T Program of Hebei (18277795D).

\section{Disclosure}

The authors declare that they have no conflicts of interest for this work.

\section{References}

1. Campbell BCV, Khatri P. Stroke. Lancet. 2020;396(10244):129-142.

2. Campbell BCV, De Silva DA, Macleod MR, et al. Ischaemic stroke. Nat Rev Dis Primers. 2019;5(1):70.

3. Algra A, Wermer MJ. Stroke in 2016: stroke is treatable, but prevention is the key. Nat Rev Neurol. 2017;13(2):78-79.

4. Bartoli F, Pompili M, Lillia N, et al. Rates and correlates of suicidal ideation among stroke survivors: a meta-analysis. J Neurol Neurosurg Psychiatry. 2017;88(6):498-504.

5. Cai W, Mueller C, Li YJ, Shen WD, Stewart R. Post stroke depression and risk of stroke recurrence and mortality: a systematic review and meta-analysis. Ageing Res Rev. 2019;50:102-109.

6. Whyte EM, Mulsant BH. Post stroke depression: epidemiology, pathophysiology, and biological treatment. Biol Psychiatry. 2002;52 (3):253-264.

7. Das J, K R G. Post stroke depression: the sequelae of cerebral stroke. Neurosci Biobehav Rev. 2018;90:104-114.

8. Dhir A, Kulkarni SK. Involvement of L-arginine-nitric oxide-cyclic guanosine monophosphate pathway in the antidepressant-like effect of venlafaxine in mice. Prog Neuropsychopharmacol Biol Psychiatry. 2007;31(4):921-925.

9. Zhang J, Snyder SH. Nitric oxide in the nervous system. Annu Rev Pharmacol Toxicol. 1995;35:213-233.

10. Gałecki P, Talarowska M. Inflammatory theory of depression. Teoria zapalna depresji - najważniejsze fakty. Psychiatr Pol. 2018;52 (3):437-447.

11. Lu DY, Leung YM, Su KP. Interferon- $\alpha$ induces nitric oxide synthase expression and haem oxygenase-1 down-regulation in microglia: implications of cellular mechanism of IFN- $\alpha$-induced depression. Int J Neuropsychopharmacol. 2013;16(2):433-444.

12. Dhir A, Kulkarni SK. Involvement of nitric oxide (NO) signaling pathway in the antidepressant action of bupropion, a dopamine reuptake inhibitor. Eur J Pharmacol. 2007;568(1-3):177-185.

13. Wang D, An SC, Zhang X. Prevention of chronic stress-induced depression-like behavior by inducible nitric oxide inhibitor. Neurosci Lett. 2008;433(1):59-64.

14. Park BK, Kim NS, Kim YR, et al. Antidepressant and anti-neuroinflammatory effects of Bangpungtongsung-San. Front Pharmacol. 2020;11:958.

15. Zhou XY, Zhang F, Ying CJ, et al. Inhibition of iNOS alleviates cognitive deficits and depression in diabetic mice through downregulating the NO/sGC/cGMP/PKG signal pathway. Behav Brain Res. 2017;322(Pt A):70-82.

16. Miyazaki D, Nakamura T, Toda M, Cheung-Chau KW, Richardson RM, Ono SJ. Macrophage inflammatory protein-1alpha as a costimulatory signal for mast cell-mediated immediate hypersensitivity reactions. J Clin Invest. 2005;115(2):434-442.

17. Merendino RA, Di Pasquale G, De Luca F, et al. Involvement of fractalkine and macrophage inflammatory protein-1 alpha in moderate-severe depression. Mediators Inflamm. 2004;13(3):205-207.

18. Ajuebor MN, Virág L, Flower RJ, Perretti M, Szabó C. Role of inducible nitric oxide synthase in the regulation of neutrophil migration in zymosan-induced inflammation. Immunology. 1998;95 (4):625-630.
19. Mühl H, Dinarello CA. Macrophage inflammatory protein-1 alpha production in lipopolysaccharide-stimulated human adherent blood mononuclear cells is inhibited by the nitric oxide synthase inhibitor N(G)-monomethyl-L-arginine. J Immunol. 1997;159(10):5063-5069.

20. Kwah LK, Diong J. National Institutes of Health Stroke Scale (NIHSS). J Physiother. 2014;60(1):61.

21. Stein DJ, Phillips KA, Bolton D, Fulford KW, Sadler JZ, Kendler KS. What is a mental/psychiatric disorder? From DSM-IV to DSM-V. Psychol Med. 2010;40(11):1759-1765.

22. Zimmerman M, Martinez JH, Young D, Chelminski I, Dalrymple K. Severity classification on the Hamilton Depression Rating Scale. $J$ Affect Disord. 2013;150(2):384-388.

23. Kumar S. Sobering news about post-stroke depression. Lancet Psychiatry. 2017;4(1):2-3.

24. Swardfager W, Winer DA, Herrmann N, Winer S, Lanctôt KL. Interleukin-17 in post-stroke neurodegeneration. Neurosci Biobehav Rev. 2013;37(3):436-447.

25. Guix FX, Uribesalgo I, Coma M, Muñoz FJ. The physiology and pathophysiology of nitric oxide in the brain. Prog Neurobiol. 2005;76 (2):126-152.

26. Geppert AM, Losy J, Przedpelska-Ober E, Kozubski W. CCL3 correlates with the number of mood disturbances and personality changes in patients with Alzheimer's disease. Psychiatry Res. 2010;176(2-3):261-264.

27. Herken H, Gurel A, Selek S, et al. Adenosine deaminase, nitric oxide, superoxide dismutase, and xanthine oxidase in patients with major depression: impact of antidepressant treatment. Arch Med Res. 2007;38(2):247-252.

28. Dhir A, Kulkarni SK. Nitric oxide and major depression. Nitric Oxide. 2011;24(3):125-131.

29. Cepeda MS, Stang P, Makadia R. Depression Is associated with high levels of C-reactive protein and low levels of fractional exhaled nitric oxide: results from the 2007-2012 national health and nutrition examination surveys. J Clin Psychiatry. 2016;77(12):1666-1671.

30. Kudlow P, Cha DS, Carvalho AF, McIntyre RS. Nitric oxide and major depressive disorder: pathophysiology and treatment implications. Curr Mol Med. 2016;16(2):206-215.

31. Hashioka S, Klegeris A, Monji A, et al. Antidepressants inhibit interferon-gamma-induced microglial production of IL-6 and nitric oxide. Exp Neurol. 2007;206(1):33-42.

32. Lindqvist D, Hall S, Surova Y, et al. Cerebrospinal fluid inflammatory markers in Parkinson's disease-associations with depression, fatigue, and cognitive impairment. Brain Behav Immun. 2013;33:183-189.

33. Dowlati Y, Herrmann N, Swardfager W, et al. A meta-analysis of cytokines in major depression. Biol Psychiatry. 2010;67(5):446-457.

34. Sperner-Unterweger B, Kohl C, Fuchs D. Immune changes and neurotransmitters: possible interactions in depression? Prog Neuropsychopharmacol Biol Psychiatry. 2014;48:268-276.

35. Pang L, Ye W, Che XM, Roessler BJ, Betz AL, Yang GY. Reduction of inflammatory response in the mouse brain with adenoviral-mediated transforming growth factor-ss1 expression. Stroke. 2001;32(2):544-552.

36. Cowell RM, Xu H, Galasso JM, Silverstein FS. Hypoxic-ischemic injury induces macrophage inflammatory protein-1alpha expression in immature rat brain. Stroke. 2002;33(3):795-801.

37. Ogłodek EA. Changes in the concentrations of inflammatory and oxidative status biomediators (MIP- $1 \alpha$, PMN elastase, MDA, and IL-12) in depressed patients with and without posttraumatic stress disorder. Pharmacol Rep. 2018;70(1):110-118.

38. Simon NM, McNamara K, Chow CW, et al. A detailed examination of cytokine abnormalities in major depressive disorder. Eur Neuropsychopharmacol. 2008;18(3):230-233.

39. Ferrari F, Villa RF. The neurobiology of depression: an integrated overview from biological theories to clinical evidence. Mol Neurobiol. 2017;54(7):4847-4865. 
40. Yang L, Zhang Z, Sun D, et al. Low serum BDNF may indicate the development of PSD in patients with acute ischemic stroke. Int J Geriatr Psychiatry. 2011;26(5):495-502.

41. Terroni L, Amaro E, Iosifescu DV, et al. Stroke lesion in cortical neural circuits and post-stroke incidence of major depressive episode: a 4-month prospective study. World J Biol Psychiatry. 2011;12 (7):539-548.
42. Suarez EC, Krishnan RR, Lewis JG. The relation of severity of depressive symptoms to monocyte-associated proinflammatory cytokines and chemokines in apparently healthy men. Psychosom Med. 2003;65(3):362-368

\section{Publish your work in this journal}

Neuropsychiatric Disease and Treatment is an international, peerreviewed journal of clinical therapeutics and pharmacology focusing on concise rapid reporting of clinical or pre-clinical studies on a range of neuropsychiatric and neurological disorders. This journal is indexed on PubMed Central, the 'PsycINFO' database and CAS, and is the official journal of The International Neuropsychiatric Association (INA). The manuscript management system is completely online and includes a very quick and fair peer-review system, which is all easy to use. Visit http://www.dovepress.com/testimonials.php to read real quotes from published authors.

Submit your manuscript here: https://www.dovepress.com/neuropsychiatric-disease-and-treatment-journal 